\title{
Hydrogen Induced Contact Resistance in PERC Solar Cells
}

\author{
Phillip Hamer ${ }^{1,2}$, Catherine Chan ${ }^{2}$, Ruy S Bonilla ${ }^{1}$, Brett Hallam², Gabrielle Bourret-Sicotte ${ }^{1}$, \\ Katherine A Collett ${ }^{1}$, Stuart Wenham ${ }^{2}$ and Peter R Wilshaw ${ }^{1}$ \\ ${ }^{1}$ Department of Materials, University of Oxford, Oxford, OX1 3PH, United Kingdom \\ ${ }^{2}$ School of Photovoltaic and Renewable Energy Engineering, University of New South Wales, \\ Sydney, NSW, 2052, Australia
}

Keywords: Crystalline Silicon, Hydrogen, Photovoltaics, Solar Cells, Degradation, Contact Resistance.

\section{Abstract}

The origins of an increase in the series resistance of PERC multicrystalline silicon solar cells due to postfiring thermal processes are investigated. This effect has been shown to be capable of reducing the fill factor of finished cells by up to $20 \%_{\mathrm{ABS}}$, severely degrading their performance. It is observed that electric currents applied either during or after these thermal processes can greatly alter the series resistance, either causing $\mathrm{R}_{\mathrm{S}}$ to increase by more than an order of magnitude or suppressing the effect entirely. It is demonstrated that this behavior is in good agreement with the expected interactions of hydrogen with dopants and electric fields within silicon wafers. It is therefore speculated that at least part of the observed increase in resistance is due to the motion of hydrogen within the cell itself.

\section{Introduction}

There has been significant recent interest in post co-firing processes for silicon solar cells. These processes are often intended to getter or improve passivation of defects [1-4], or to mitigate degradation effects [5-8]. While these approaches have proven to be effective in improving the bulk lifetime and longterm stability of devices, an unintended consequence has been a reported increase in device series resistance $\left(\mathrm{R}_{\mathrm{S}}\right)[5,9,10]$.

Initial observations of this effect on heavily diffused emitters concluded that the increased $\mathrm{R}_{\mathrm{S}}$ was the result of a thickening of the glass layer surrounding silver crystallites at the Ag-Si interface [9]. However, more recently the authors have reported an unstable component to the $\mathrm{R}_{\mathrm{S}}$ increase that appears to be related to the motion of charged particles [10]. This unstable component has been shown to be most significant at temperatures between 450 and $500^{\circ} \mathrm{C}$, while a more permanent increase in $\mathrm{R}_{\mathrm{S}}$ is observed at higher temperatures. Further investigation has revealed that this increase in $\mathrm{R}_{\mathrm{S}}$ is almost exclusively due to an increase in the contact resistance at the interface between the screen-printed silver fingers and the silicon surface $[9,10]$.

It has been suggested that this unstable increase in contact resistance is related to the motion of hydrogen [10]. This is in good agreement with recent simulations on the re-distribution of hydrogen at similar temperatures [11] which predicts that there will be a significant build-up of hydrogen at the metal contacts. The mechanism by which hydrogen might act to increase contact resistance is currently unknown, although possibilities include alteration of the glass between the screen printed silver and the silicon or through interaction with defects and silver crystallites underlying this glass layer [12-15].

This paper will further explore the phenomenon of increased contact resistance due to post-firing thermal processes. It is demonstrated how electric fields within the cells can be used to enhance or suppress this change in $\mathrm{R}_{\mathrm{S}}$. The observed changes in $\mathrm{R}_{\mathrm{S}}$ and the expected behavior of hydrogen in solar cells is then 
compared [11]. It is found that there is a strong correlation between the experimentally observed increase in $\mathrm{R}_{\mathrm{S}}$ and the simulated build up of hydrogen at the surface.

\section{Methods and Materials}

Full size $156 \times 156 \mathrm{~mm}$ PERC cells were fabricated on an industrial manufacturing line. The wafers used were p-type boron-doped $1.2 \Omega \mathrm{cm}$ high-performance (HP) mc-Si wafers. The wafers underwent the following standard treatments: acidic texturing (final thickness $180 \mu \mathrm{m}$ ), cleaning, $\mathrm{POCl}_{3}$ diffusion $\left(90 \Omega \mathrm{sq}^{-1}\right)$, rear-side etch, and finally plasma-enhanced chemical vapor deposition (PECVD). The layers deposited by PECVD were hydrogenated silicon nitride ( $\left.\mathrm{SiN}_{X}: \mathrm{H}\right)$ with a thickness of $75 \mathrm{~nm}$ and refractive index of 2.1 on the front n-type diffused side and a hydrogenated aluminium oxide $\left(\mathrm{AlO}_{\mathrm{x}}: \mathrm{H}\right) / \mathrm{SiN}_{\mathrm{X}}: \mathrm{H}$ stack with thicknesses of 10 and $100 \mathrm{~nm}$, respectively on the rear p-type side. Point openings in this stack were formed using laser ablation, and the wafers were then screen printed with aluminium paste over the entire rear surface and silver paste on the front in a grid pattern. This was followed by a standard drying and cofiring step in an industrial belt-furnace to form the metal contacts and release hydrogen from the dielectric layers for bulk and surface passivation [10]. The wafers were then cleaved into smaller samples for further processing.

Whereas in previous work [10] samples have been re-fired using a belt furnace, in this work samples were annealed on a heated stage in the dark as shown in Figure 1. The aluminium stage was PID temperature controlled via Labview and a mechanically affixed thermocouple as shown in Figure 1. The probes were connected to a Keithley 2401 source measuring unit (SMU) to allow for the application of external bias to the samples and for in-situ observation of I-V characteristics. The samples were I-V tested at room temperature using the same equipment.

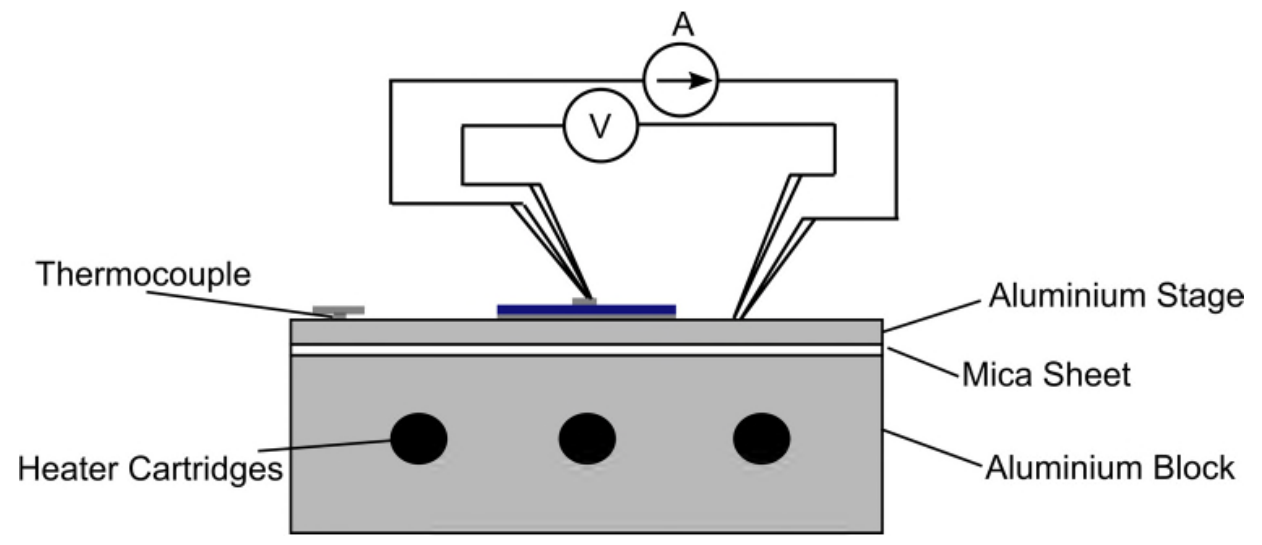

Figure 1: Experimental set-up for thermal processing and in-situ monitoring of I-V characteristics for solar cell samples.

Room temperature characterization of $\mathrm{R}_{\mathrm{S}}$ was done through profiling the dark I-V curve and subsequently fitting using a two diode model $[16,17]$. At elevated temperature, the change in series resistance $\left(\Delta \mathrm{R}_{\mathrm{S} .350}\right)$ was evaluated by plotting the initial (lowest resistance) voltage as a function of current (V-I plot). This plot was then interpolated and subtracted from subsequent V-I measurements as shown in Figure 2. The average values and standard deviation of the change in series resistance was then calculated using the positive current values of the resulting curves using:

$$
\Delta R_{S .350}=\frac{V_{\text {meas }}-V_{\text {initial }}}{I}
$$


It is important to note that in some instances $\Delta \mathrm{R}_{\mathrm{S} .350}$ changed so rapidly that there were alterations during the time interval $(0.7 \mathrm{~s})$ for the measurement to be carried out. This not only introduced errors into the measurements themselves but meant that altering the measurement frequency lead to slight changes in behavior over the entire process. In order to keep this effect manageable measurements were performed every 5 minutes during the process.
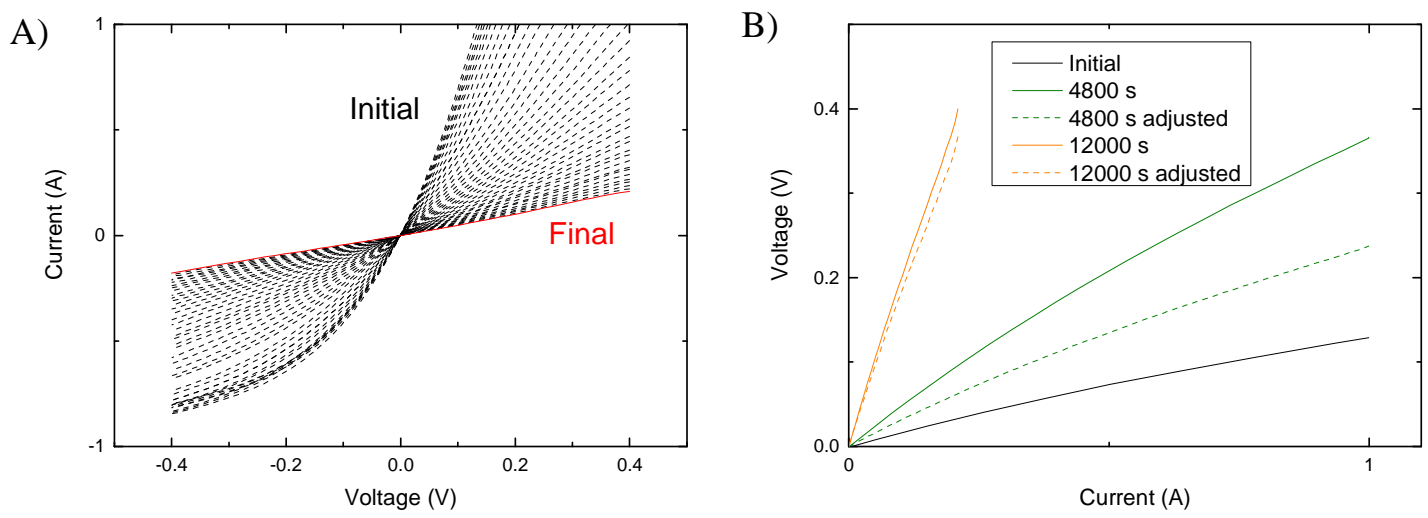

Figure 2: A) In-situ I-V curves of a sample annealed at $350^{\circ} \mathrm{C}$ with a $0.2 \mathrm{~V}$ forward bias for 4 hours sampled at 5 minute intervals. B) V-I curves at the start of annealing, after $4800 \mathrm{~s}$ and $12000 \mathrm{~s}$ and curves adjusted by subtracting the initial curve.

In order to illustrate the effect of the increase in series resistance simple simulations were carried out using PC1D [18], with the results for cell fill factor and efficiency presented in Figure 3. The initial average $\mathrm{R}_{\mathrm{S}}$ value for our samples of $0.69 \Omega . \mathrm{cm}^{2}$ reduces the fill factor and efficiency by less than $5 \%_{\text {relative }}$ compared to the case with no series resistance, while an increase of $R_{S}$ to $2 \Omega . \mathrm{cm}^{2}$ results in a $14 \%_{\text {relative }}$ reduction and an increase to $10 \Omega . \mathrm{cm}^{2}$ results in a loss of more than $50 \%$ relative. The parameters for these simulations may be found in Appendix A.

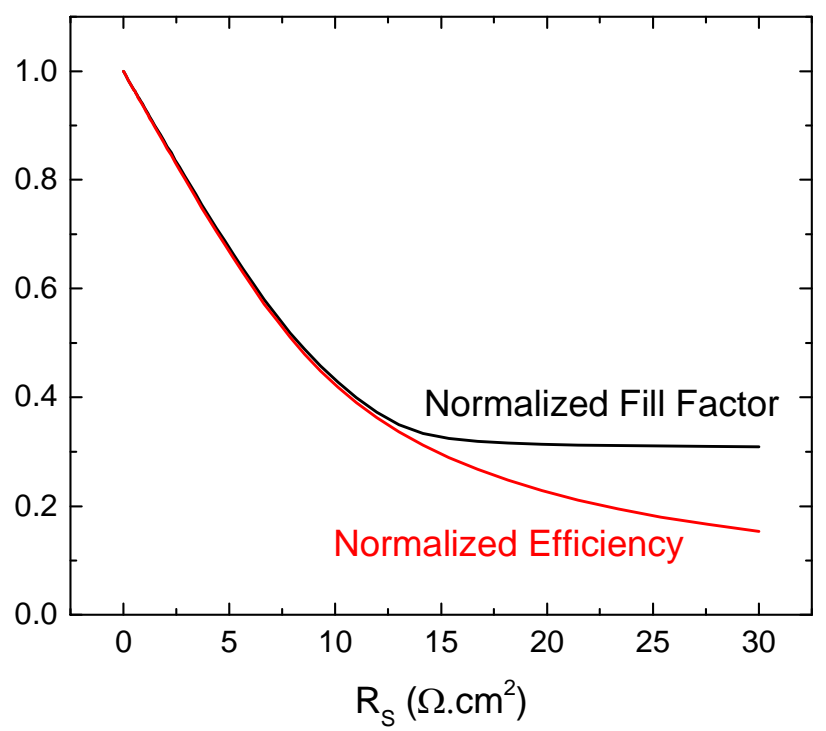

Figure 3: Normalized Fill Factor and Power Conversion Efficiency as simulated using PCID as a function of series resistance. 


\section{Results}

\section{Transmission Line Measurements}

In order to accurately observe $\Delta \mathrm{R}_{\mathrm{S} .350}$ using in-situ measurements temperatures close to $350^{\circ} \mathrm{C}$ were used in this work, resulting in a longer timescale for the $\Delta \mathrm{R}_{\mathrm{S} .350}$ increase. It was therefore important to investigate whether the effects observed behaved in the same way as those reported in previous work [10]. In particular, it was necessary to check that increases in $\mathrm{R}_{\mathrm{S}}$ were caused by a change in the contact resistance at the screen printed metal fingers and that this increase was unstable under applied current at room temperature. This was done using transmission line measurements (TLM) [19] between the silver fingers of $3 \times 2.2 \mathrm{~cm}$ samples cleaved from multi PERC cells without a bus bar. As in previous work, in order to stabilize the readings one of the contacts had its resistance minimized using a forward bias current prior to the measurement [10].

Figure 4 presents TLM results of samples annealed at $350^{\circ} \mathrm{C}$ for $2 \mathrm{~h}$. After the anneal currents of $1 \mathrm{~mA}$ reverse bias, and then $1 \mathrm{~mA}, 5 \mathrm{~mA}, 25 \mathrm{~mA}$ and finally $50 \mathrm{~mA}$ forward bias were applied between the probes for $120 \mathrm{~s}$ each at room temperature. Figure $3 \mathrm{~B}$ ) shows the evolution of the measured resistance during these processes, with each successive increase in applied current demonstrating an initial rapid drop in resistance followed by the establishment of an apparent near-equilibrium. For both the initial measurement and measurements after forward bias currents in excess of $5 \mathrm{~mA}$ had been applied an excellent straight-line fit was obtained. In contrast, the measurements taken immediately after the smallest currents had been applied showed much greater variation. The significant change in the intercept of these curves indicates that the primary effect was an increase in the contact resistance, which in this case increased by more than an order of magnitude post-anneal.

The fits presented in Table 1 after $1 \mathrm{~mA}$ reverse and forward bias current, and to a lesser extent after 5 $\mathrm{mA}$ excluding the first point, show a slight increase in gradient. This would usually indicate a change in sheet resistance of the emitter, however it is considered unlikely that this occurred in these experiments. While there may be some effects that could de-activate phosphorous in the emitter during annealing, it is not plausible that they could have been reversed so easily at room temperature. This effect is therefore presently attributed to local variations in contact resistance across the wafer and between measurements. This is supported by the results after forward bias annealing with higher currents. It is important to note that while hydrogen deactivation across the whole area between metal fingers is rejected, this does not exclude the possibility that there is significant de-activation of phosphorous in the region immediately underneath the metal contacts.

The behavior is almost identical to that observed in our earlier work [10] for samples re-fired at temperatures between $400-600^{\circ} \mathrm{C}$, making it extremely likely that this is the same effect, albeit with a reduced rate due to the lower temperatures. Again, the primary influence of the thermal treatment has been an increase in the contact resistance between the screen-printed silver fingers and the silicon emitter, which is unstable at room temperature under an applied current. As in previous work, it was also observed that the resistance began to increase at room temperature once the forward bias was removed. 

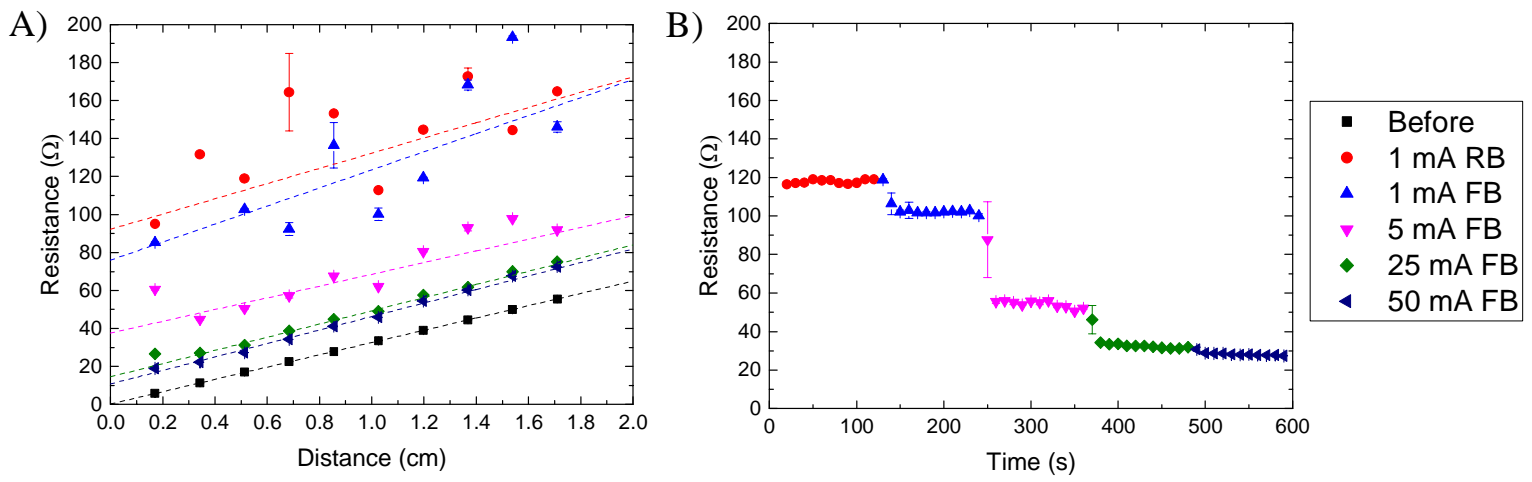

Figure 4:A) TLM measurements and fits after firing (before), after post-firing annealing in the dark at $353^{\circ} C$ for 2 hours and after subsequent applications of currents of $1 \mathrm{~mA}$ reverse bias $(1 \mathrm{~mA} \mathrm{RB})$ and $1,5,25$ and $50 \mathrm{~mA}$ forward bias (FB) for $120 \mathrm{~s}$. B) Resistance measurements for a finger spacing of $0.52 \mathrm{~cm}$ ( $3^{\text {rd }}$ point) during each bias application.

Table 1: Intercepts and gradients for the fits to the data in Figure $3 \mathrm{~A}$

\begin{tabular}{lcccccc} 
& Before & 1 $\mathbf{~ m A ~ R B}$ & $\mathbf{1} \mathbf{~ m A ~ F B}$ & $\mathbf{5} \mathbf{~ m A ~ F B}$ & $\mathbf{2 5} \mathbf{~ m A ~ F B}$ & $\mathbf{5 0} \mathbf{~ m A ~ F B}$ \\
\hline Intercept & $0.28 \pm 0.02$ & $92 \pm 5$ & $76 \pm 12$ & $38 \pm 7$ & $14.6 \pm 1.0$ & $10.8 \pm 0.9$ \\
Gradient & $32.34 \pm 0.07$ & $40 \pm 8$ & $48 \pm 13$ & $31 \pm 7$ & $34.7 \pm 1.2$ & $35.5 \pm 1.3$
\end{tabular}

\section{Annealing under a Current-Controlled Bias}

The instability of $\mathrm{R}_{\mathrm{S}}$ at room temperature under applied current indicates that this effect may be altered by an applied current during annealing. In order to obtain more information on the nature of this effect, anneals were carried out at elevated temperature with a current-voltage source applying up to $1 \mathrm{~A}$, voltage limited to $20 \mathrm{~V}$ in forward and reverse bias. The samples used were $30 \times 20 \mathrm{~mm}$ samples cleaved with a busbar in the centre to allow good contact to be made to the front metal grid.

Figure 5 presents the extracted $\mathrm{R}_{\mathrm{S}}$, as measured at room temperature, after 2 hour anneals at a range of temperatures. It is clear that the extent to which $R_{S}$ increased was greater at higher temperatures within this range, and that applied current had a significant effect upon the process. Under forward current, the observed RS was comparable, or even slightly lower, than for those with no current at temperatures below $300^{\circ} \mathrm{C}$. For temperatures above $300^{\circ} \mathrm{C} \mathrm{R}_{\mathrm{S}}$ after annealing with forward current began to increase rapidly with temperature, and was significantly worse than that with no applied current at temperatures above $350^{\circ} \mathrm{C}$. In contrast, $R_{S}$ after annealing with a reverse current at temperatures above $300^{\circ} \mathrm{C}$ was significantly lower than that after annealing with either forward current or no current. Indeed, there was minimal increase in $\mathrm{R}_{\mathrm{S}}$ over the value of $0.69 \pm 0.11 \Omega . \mathrm{cm}^{2}$ observed before annealing.

Perhaps the most surprising aspect of these results is the sharp departure of the behavior at elevated temperatures from that at room temperature under applied current. At room temperature, an applied forward current acts to reverse the increase in $R_{S}$, wheras at elevated temperatures the increase in $R_{S}$ is much more severe when a forward current is present. In contrast, applying a reverse current at high temperatures appears to suppress the $R_{S}$ increase, while negative bias at room temperature has not been observed to have any significant impact. This leads to the supposition that there may be more than one effect at work when current/bias is applied to the cells. 


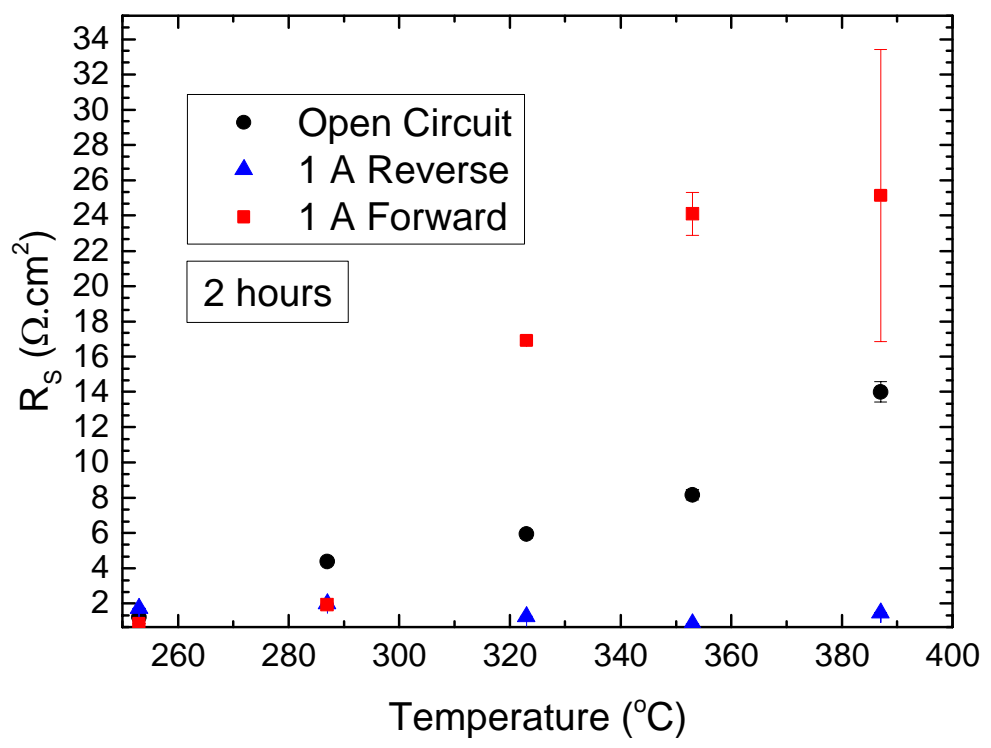

Figure 5: $R_{S}$ as measured at room temperature after annealing in the dark for 2 hours at temperatures between $250^{\circ} \mathrm{C}$ and $400^{\circ} \mathrm{C}$, either at open circuit (black circles), under 1 A forward bias (red squares) or under 1 A reverse bias. The sample processed under forward bias at the highest temperature showed very significant instability during room temperature measurements which caused the large uncertainties in the measured values. Samples with no anneal had $R_{S}$ values of $0.69 \pm 0.11 \Omega . \mathrm{cm}^{2}$..

\section{In-Situ Measurement with Voltage-Controlled Bias}

In order to investigate the effect of an applied bias in more detail a second set of $30 \times 30 \mathrm{~mm}$ samples with busbars were annealed under voltages of -0.2 to $0.2 \mathrm{~V}$ in the dark at $350^{\circ} \mathrm{C}$, with in-situ measurements every 5 minutes. The observed $\Delta \mathrm{R}_{\mathrm{S} .350}$ is shown in Figure 6. Here, it is demonstrated that under a forward bias the increase in contact resistance is greatly accelerated while even a $0.2 \mathrm{~V}$ reverse bias is enough to almost completely suppress any change in contact resistance. Again, there is no obvious upper limit for the contact resistance. As has been previously observed, a $120 \mathrm{~s}, 1 \mathrm{~A}$ forward bias treatment applied at room temperature is capable of reversing much of this increase in contact resistance.

One point of interest is that the difference between samples annealed with no bias and those subjected to a forward bias anneal was much more significant in the results presented in Figure 5 when compared with those in Figure 5. While not enough samples have been processed to date to rule out variation between samples, it is also possible that the lower forward bias currents involved in maintaining $0.2 \mathrm{~V}$ across the structure (once $\Delta \mathrm{R}_{\mathrm{S} .350}$ had begun to increase) mean that the current through the device was significantly reduced. This provides further evidence that there is likely more than one effect involved in this phenomenon, one that is dependent on the voltage across the n-p junction and another related to either the current or the excess carrier density within the sample. 
A)

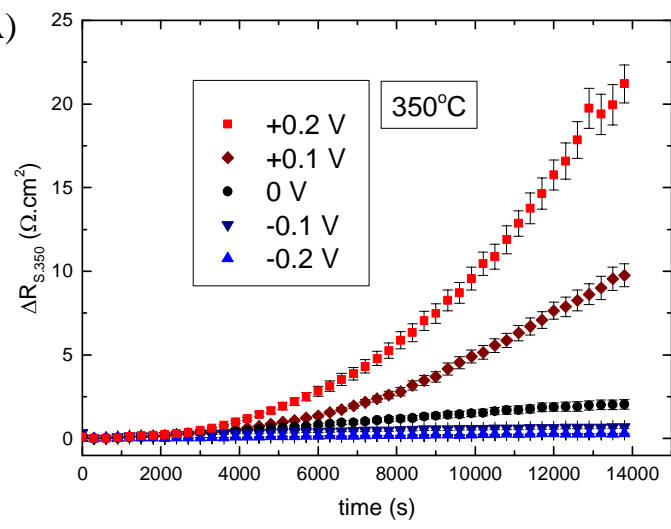

B)

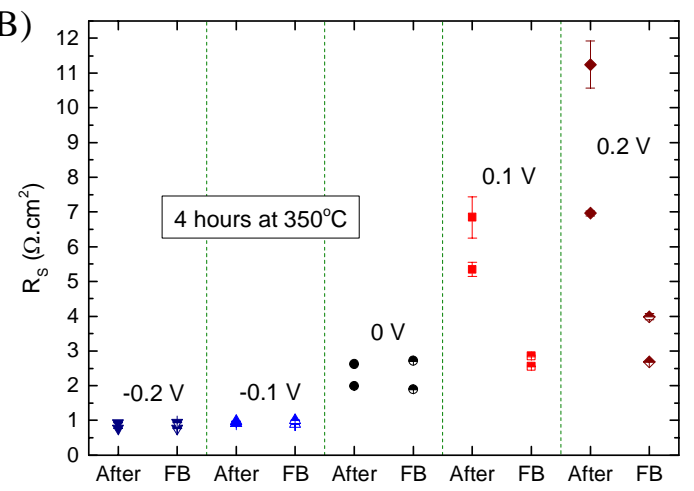

Figure 6: A) In-situ $\Delta R_{S .350}$ observed during a 4 hour annealed in the dark at $350^{\circ} \mathrm{C}$ for samples with an applied bias between0.2 and 0.2 V. B) Room temperature measurement of $R_{S}$ for samples subjected to 4 hour anneals as in A) (after), and with subsequent application of 1 A forward bias for $120 \mathrm{~s}$ (FB).

4. Reversibility of series resistance increase at elevated temperatures

One of the signature traits that a process within a silicon solar cell involves hydrogen is the reversibility of that process $[1,20]$. Given the clear influence of an applied bias at $350^{\circ} \mathrm{C}$ it was logical to investigate how reversing this bias would affect the behavior of the contact resistance. Samples were selected that had previously been subjected to a 4 hour anneal under either $0.2 \mathrm{~V}$ forward bias, $0.2 \mathrm{~V}$ reverse bias or $0 \mathrm{~V}$ bias, as shown in Figure 6, and subsequently forward biased at room temperature to minimize the contact resistance. These samples were then subjected to a 65 minute anneal at $350^{\circ} \mathrm{C}$ with an applied bias of 0.2 $\mathrm{V}$ followed immediately by a 65 minute anneal with the bias reversed, with the $\Delta \mathrm{R}_{\mathrm{S} .350}$ presented in Figure 6 .

There are two important observations from these results. Firstly, the effect is almost completely reversible, while $0.2 \mathrm{~V}$ forward bias leads to an increase in $\Delta \mathrm{R}_{\mathrm{S} .350}$, applying $-0.2 \mathrm{~V}$ rapidly returns $\Delta \mathrm{R}_{\mathrm{S} .350}$ towards zero. Subsequent investigations confirmed that this could be repeated several times with the same general behavior repeated. This high degree of reversibility strongly implies that hydrogen is playing a role in the observed contact resistance changes.

Secondly, the prior processing had a significant impact on the rate at which $\Delta \mathrm{R}_{\mathrm{S}}$ increased under forward bias. The sample that had previously been annealed with a forward bias showed a much more rapid increase than had been observed in Figure 6, and also a greater rate than samples which had previously been annealed with either no bias or reverse biased. The sample that had previously been annealed without bias also showed a $\Delta \mathrm{R}_{\mathrm{S} .350}$ increase more rapid than for the samples with no prior post-firing processing. However, the rate of increase for the sample that had previously been reverse biased was much closer, albeit still slightly higher, than that of a sample with no prior post-firing thermal treatments.

In contrast, the effect of a forward bias current at room temperature only temporarily reduces $\mathrm{R}_{\mathrm{S}}$ and has no significant impact on subsequent processing. This observation provides evidence that the effects occurring during annealing under bias are on a larger scale than those which occurs under applied currents at room temperature, most likely involving transport on the scale of $\mu \mathrm{m}$ rather than $\mathrm{nm}$. This is further supported by room temperature measurements of $\mathrm{R}_{\mathrm{S}}$ of these samples at the conclusion of the process. Not only were all values close to the samples original values, but they remained stable at room temperature. This suggests that the application of a reverse bias at elevated temperatures could provide a (relatively) rapid means of mitigating an increase in contact resistance due to post-firing processing. 


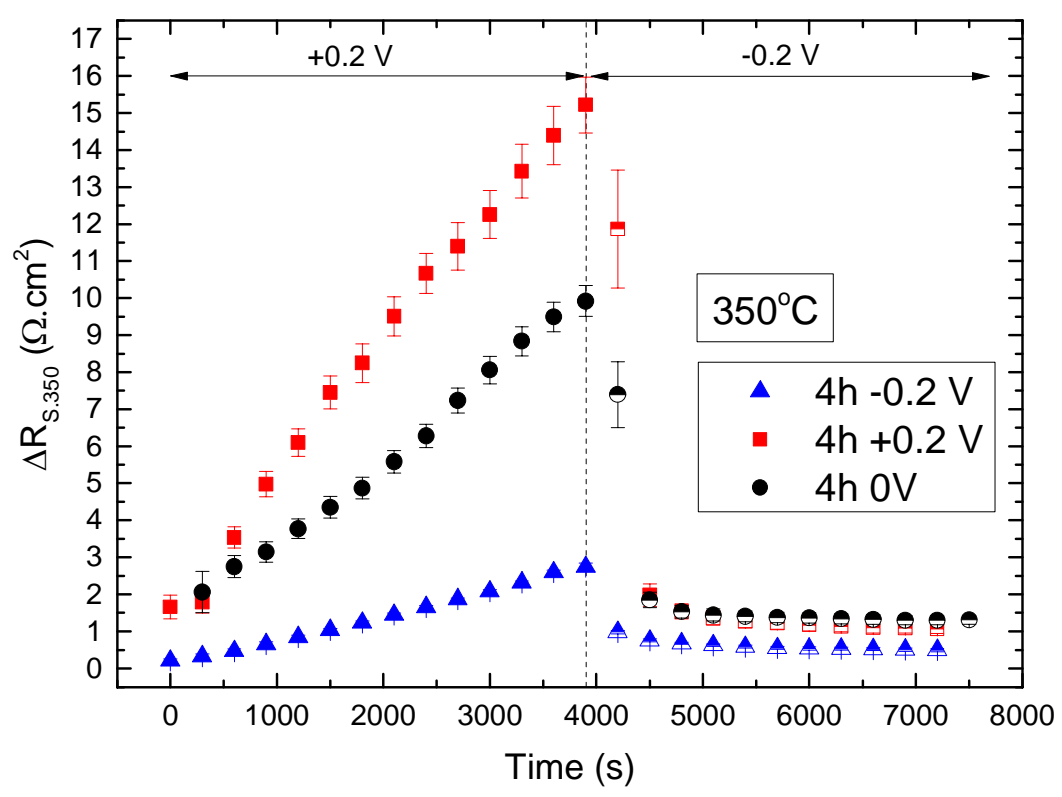

Figure 7: In-situ changes in $\Delta R$ s.350 observed during 65 minute annealing in the dark at $350^{\circ} \mathrm{C}$ with $0.2 \mathrm{~V}$ forward bias applied, immediately followed by the same process with the bias reversed. Samples had previously been annealed for 4 hours at $350^{\circ} \mathrm{C}$ with either $0.2 \mathrm{~V}$ forward bias (red squares), $0 \mathrm{~V}$ (black circles) or $0.2 \mathrm{~V}$ reverse bias (blue triangles), and then had $1 \mathrm{~A}$ forward current applied at room temperature for $120 \mathrm{~s}$.

\section{Discussion}

Both the reversibility of $\Delta \mathrm{R}_{S .350}$ and the susceptibility to electric fields lead to the conclusion that this effect is related to the motion of charged particles. Given the ability to alter the behavior at room temperature these particles must be highly mobile and found in significant concentrations in PERC solar cells. The most obvious candidate to explain these effects is hydrogen. This has also been suggested in previous work [10].

The behavior under electric field may then be analyzed in terms of the motion of hydrogen within a silicon solar cell. In a recent paper the authors presented a simplified model for the transport of hydrogen across a $p-n$ junction, including simulations of the re-distribution of hydrogen during thermal processing [11]. The model is not yet capable of handling non-equilibrium conditions, however, if it is assumed that hydrogen within the structure does not significantly alter carrier concentration profiles and electric fields it is possible to carry out simulations using profiles generated in an external solver, for example PC1D [18].

Figure 8 presents the results of such simulations run at a temperature of $350^{\circ} \mathrm{C}$, with an initially uniform concentration of interstitial hydrogen of $1 \times 10^{15}$ atoms $/ \mathrm{cm}^{3}$ and an example doping profile from [21]. To simplify the calculations, dimer formation and dissociation were not included in these simulations, meaning that hydrogen could only exist in interstitial form or bound to dopant ions. As expected, the primary effect of the applied bias was to alter the electric field observed across the junction with a reverse bias leading to a much stronger electric field penetrating deeper into the structure. This leads to enormous differences in the re-distribution of hydrogen across these structures, as observed in Figure 8 B) and C).

As with previous simulations using this model [11] the critical point of interest is the "cross-over" where the majority of hydrogen changes from being in the negative to the positive charge state. On the surface side of this point hydrogen in the negative charge state will be rapidly swept towards the surface of the 
structure by the electric field due to the electrically active phosphorous profile, which is largely unchanged by the applied bias. The hydrogen profile in this region will thus rapidly approach a local equilibrium resulting in a concentration profile such that there is minimal movement of $\mathrm{H}$ - under the action of the electric field (drift term) and concentration gradient (diffusion term). Hydrogen in this region will also be in local equilibrium with hydrogen bound to phosphorous ions. Because hydrogen is so susceptible to electric fields this results in a sharp decrease in $\mathrm{H}^{-}$concentration from the surface to the cross-over point. This is mirrored with $\mathrm{H}^{+}$on the other side of the cross-over point, however, in this case the electric field varies significantly with applied bias.

In the case where a forward bias is applied, the electric field on the bulk side of the cross-over point is relatively weak. Only a shallow concentration gradient of $\mathrm{H}^{+}$towards the cross-over point is required for a significant flux of hydrogen into the emitter. This flux is driven by the transport of $\mathrm{H}^{+}$to the cross over point and by the transport of $\mathrm{H}^{-}$from the cross-over point towards the surface as shown in Figure $8 \mathrm{~B}$ ). This leads to a long-range redistribution with simulations showing the majority of hydrogen in the first $20 \mu \mathrm{m}$ of the structure moving towards the emitter region and surface hydrogen concentrations exceeding $10^{17}$ atoms $/ \mathrm{cm}^{3}$. In contrast, where a reverse bias is applied, the stronger electric field on the bulk side of the cross-over point means that a much greater gradient in $\mathrm{H}^{+}$concentration is required for a net flux of hydrogen into the emitter from the bulk. As shown in Figure $8 \mathrm{C}$ ) this effect suppresses re-distribution of hydrogen from the bulk to the emitter.

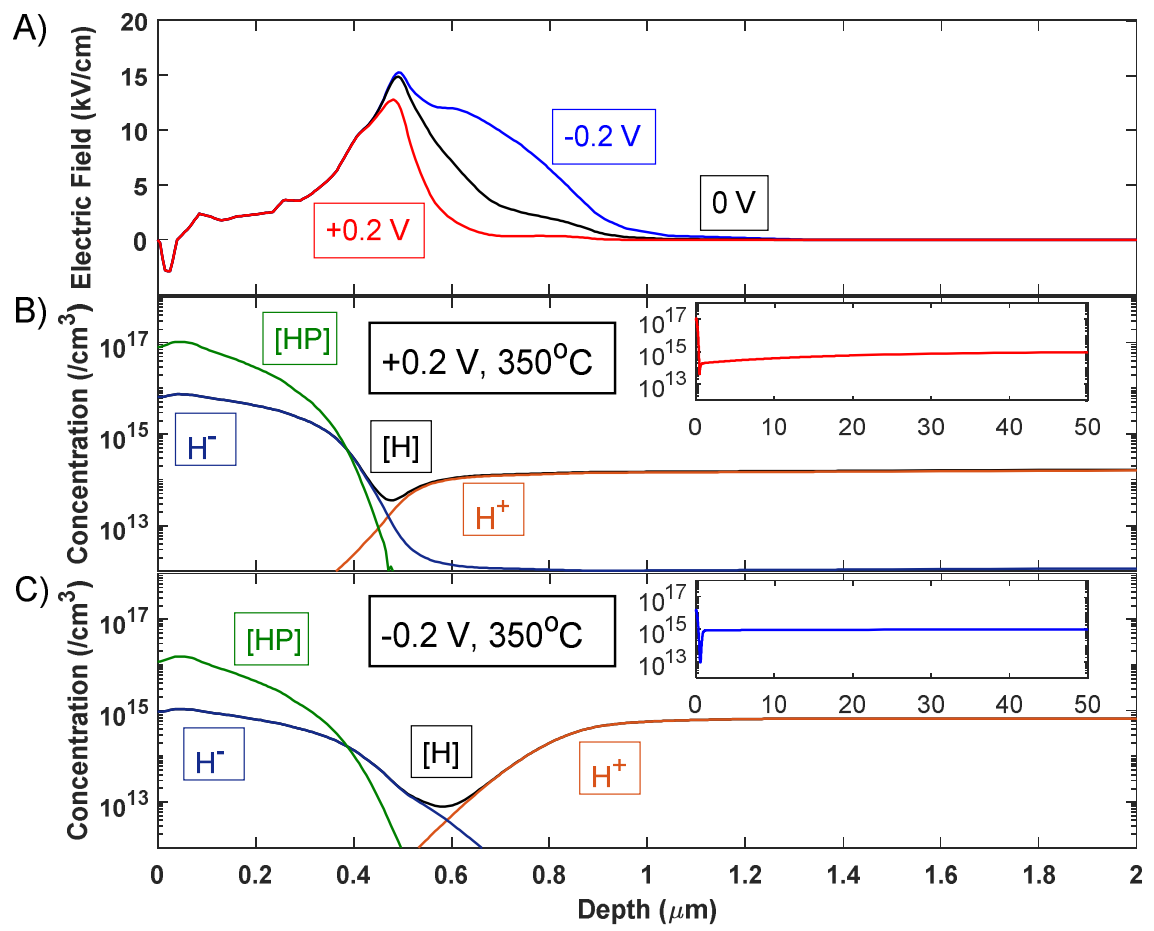

Figure 8: A) Electric fields in the first $2 \mu \mathrm{m}$ of the model as calculated using PC1D [20] with applied biases of $-0.2 \mathrm{~V}, 0 \mathrm{~V}$ and $0.2 \mathrm{~V}$ at $350^{\circ} \mathrm{C}$. B) Concentrations of hydrogen in interstitial forms $\left(\left[\mathrm{H}^{+}\right],\left[\mathrm{H}^{-}\right]\right.$and total $\left.[\mathrm{H}]=\left[\mathrm{H}^{+}\right]+\left[\mathrm{H}^{-}\right]+\left[\mathrm{H}^{0}\right]\right)$, and bound to phosphorous ions ([HP]) in the first $2 \mu \mathrm{m}$ of the model with a $0.2 \mathrm{~V}$ forward bias applied for $30 \mathrm{~s}$. Inset: Total hydrogen concentration in the first $50 \mu \mathrm{m}$. D) Concentrations of hydrogen in interstitial forms $\left(\left[\mathrm{H}^{+}\right],\left[\mathrm{H}^{-}\right]\right.$and total $\left.[\mathrm{H}]=\left[\mathrm{H}^{+}\right]+\left[\mathrm{H}^{-}\right]+\left[\mathrm{H}^{0}\right]\right)$, and bound to phosphorous ions ([HP]) in the first $2 \mu \mathrm{m}$ of the model with a $0.2 \mathrm{~V} \mathrm{reverse} \mathrm{bias} \mathrm{applied} \mathrm{for} 30 \mathrm{~s}$. Inset: Total hydrogen concentration in the first $50 \mu \mathrm{m}$. 
There is a good correlation between the behavior of hydrogen in these simulations and the experimentally observed increase in contact resistances in this work. Although the mechanism by which hydrogen affects the contact resistance is unknown, it is reasonable to assume that the extent of this effect is dependent upon the hydrogen concentration at the surface. The simulations show how a reverse bias can suppress the buildup of hydrogen in this region, while a forward bias will accelerate it, leading to a larger change in $\mathrm{R}_{\mathrm{s}}$. Furthermore, due to the high mobility of hydrogen in silicon, it is clear that the subsequent application of a different bias will rapidly redistribute hydrogen within the structure, explaining the observed reversibility in contact resistance changes. This also predicts that applying fields at high temperatures should lead to the long-range redistribution required to explain the results in Figure 7.

The effect of a forward bias current at low temperatures might also be explained in relation to hydrogen, in terms of the electric field at the contacts themselves. This electric field will be proportional to the current and the resistance at the contacts, and, by applying a forward bias, negative charges should be repelled from the contacts. As the majority of hydrogen should be in the negative charge state it would be expected that this should lead to hydrogen being swept away from the contacts. However, the hydrogen will not be pushed far from the surface, and, in agreement with the experimental results, will drift back to the surface once the applied current is removed.

Under forward bias conditions at elevated temperatures these two effects are likely to be in competition, especially in the case where a $1 \mathrm{~A}$ forward current was applied. With the more modest currents flowing during $0.2 \mathrm{~V}$ forward bias annealing at $350^{\circ} \mathrm{C}$, the long-range redistribution of hydrogen would appear to dominate over the local electric field at the contact repelling $\mathrm{H}^{-}$. Further investigation with a wider range of applied voltages may shed light on this effect, or what other mechanisms are involved.

However, there is a significant discrepancy in the timeframes required for this re-distribution to take place. Simulations carried out for just $30 \mathrm{~s}$ show significant changes in the hydrogen profiles, while $\Delta \mathrm{R}_{\mathrm{S}}$ requires, initially at least, up to hours to show a corresponding change. This is likely a consequence of the assumption within the simulations that the majority of hydrogen within the bulk is in interstitial form. In general, only a small proportion of hydrogen in the bulk will be in interstitial form at temperatures below $600^{\circ} \mathrm{C}[22,23]$, greatly reducing the rate of hydrogen re-distribution. Given the high diffusivities of interstitial hydrogen in silicon, the rate of the overall process is therefore expected to dominated by the release of interstitial hydrogen from its bound form (e.g. hydrogen dimers, hydrogen-dopant pairs or extended hydrogen complexes). However the behavior will still follow that depicted in Figure 8, since the vast majority of hydrogen transport in silicon solar cells takes place in the interstitial form [22, 24]. The dimer parameters used in previous simulations [11,22] do not match the timescale of the experimentally observed increase in $\Delta \mathrm{R}_{\mathrm{S}}$, which leads to the supposition that the majority of hydrogen in these multicrystalline samples takes other forms, with slower dissociation kinetics. Because the motion of interstitial hydrogen is so rapid, this leads to the expectation that by observing the kinetics of contact resistance changes at different temperatures, and with different p-type substrates, e.g. multi- vs mono-crystalline silicon, it may be possible to learn more about the states in which hydrogen exists in the p-type bulk of silicon solar cells.

It is also possible that the reaction kinetics of hydrogen at the contacts themselves play a role in determining the rate of the overall series resistance increase. However, this is considered unlikely given the ability to rapidly reverse the effect either at elevated temperature as in Figure 7 or even at room temperature with an applied current.

\section{Conclusion}

In this work, it has been shown that the increased contact resistance of p-type multicrystalline PERC cells with post-firing thermal processes is highly dependent upon applied currents and voltages, which can act to either accelerate or suppress the effect. It was also demonstrated that the increase is contact resistance is highly reversible with applied voltage, and can be relatively quickly recovered with the appropriate treatment. 
While the evidence for hydrogen involvement in the increased contact resistance is not yet conclusive, it has been shown that there is excellent agreement between the experimentally observed behavior and the presence of hydrogen near the metal-silicon interface, as obtained from modelling of hydrogen transport in these structures. This modelling is able to explain the observed effects upon annealing under an applied bias, as well as the high degree of reversibility in the results. This not only provides an important insight into the problem of increased contact resistance, it is potentially a very useful tool for investigating the behavior of hydrogen in silicon at temperatures between 300 and $700^{\circ} \mathrm{C}$. In particular, it may be possible to determine the kinetics of hydrogen release from complexes within the bulk, which in turn may provide better understanding of degradation mechanisms in multicrystalline silicon related to hydrogen.

The re-distribution of hydrogen from the bulk of the device to the emitter is also of particular interest given several recent reports linking hydrogen to the degradation observed in multicrystalline silicon [2528]. These include a recent report which suggested that transport of an impurity, possibly hydrogen, from the sample bulk to the surface is responsible for the regeneration of this degradation [28]. Further investigations are underway examining whether there is a correlation between increases in contact resistance and reductions in bulk degradation in these samples.

\section{Acknowledgements.}

The authors would like to thank Zijing Wang for assistance with measurements, and Pietro P. Altermatt for fruitful discussions. Work at Oxford University has been supported by the U.K. Government through the International and Industrial Engagement Fund and the Supersilicon project (EP/M024911/1), supported by the Engineering and Physical Sciences Research Council (EPSRC). Work at UNSW has been supported by the Australian Government through the Australian Renewable Energy Agency (ARENA) (ARENA 1A060 and 1-SRI001) and the Australian Centre for Advanced Photovoltaics (ACAP), and the Australian Research Council (DE170100620). The views expressed herein are not necessarily the views of the Australian Government, and the Australian Government does not accept responsibility for any information or advice contained herein. B. Hallam would like to gratefully acknowledge support from the EPSRC Supersolar I\&I fund.

\section{Appendix A: Parameters for PC1D Simulation}

The simulations in Figure 5 were carried out using PC1D5 [18]. All parameters were kept at their default value unless stated otherwise below:

\section{Device}

Device Area: $243 \mathrm{~cm}^{2}$

Front Surface Texture: Angle $=54.74^{\circ}$, Depth $=3 \mu \mathrm{m}$

Exterior Front Reflectance: 7\% fixed

Emitter Contact: Resistance set to contact resistance for each simulation

Base Contact: Resistance $=1 \times 10^{-6} \Omega$

Internal Conductor: $0.5 \mathrm{~S}$

\section{Region 1}

Thickness: $180 \mu \mathrm{m}$

P-type background doping: $1 \times 10^{16} \mathrm{~cm}^{-3}$

$1^{\text {st }}$ Front Diffusion: N-type, Erfc profile, Peak Doping $=2 \times 10^{20} \mathrm{~cm}^{-3}$, Depth Factor $=0.1 \mu \mathrm{m}$ 
$2^{\text {nd }}$ Front Diffusion: N-type, Erfc profile, Peak Doping $=1 \times 10^{19} \mathrm{~cm}^{-3}$, Depth Factor $=0.3 \mu \mathrm{m}$

Bulk Recombination: $\tau_{\mathrm{n}}=\tau_{\mathrm{p}}=100 \mu \mathrm{s}$

Front Surface Recombination: $\mathrm{J}_{0}$ model, equivalent $\mathrm{J}_{0}=20 \mathrm{fA} / \mathrm{cm}^{2}$

Rear Surface Recombination: $\mathrm{S}$ model, $\mathrm{S}_{\mathrm{n}}=\mathrm{S}_{\mathrm{p}}=124.8 \mathrm{~cm} / \mathrm{s}$

\section{References}

1. Hamer, P., et al., Manipulation of hydrogen charge states for passivation of P-type wafers in photovoltaics. IEEE Journal of Photovoltaics, 2014. 4(5): p. 1252-1260.

2. Liu, A., et al., Gettering of interstitial iron in silicon by plasma-enhanced chemical vapour deposited silicon nitride films. Journal of Applied Physics, 2016. 120(19): p. 193103.

3. Al-Amin, M. and J.D. Murphy, Increasing minority carrier lifetime in as-grown multicrystalline silicon by low temperature internal gettering. Journal of Applied Physics, 2016. 119(23): p. 235704.

4. Sun, C., F.E. Rougieux, and D. Macdonald, A unified approach to modelling the charge state of monatomic hydrogen and other defects in crystalline silicon. Journal of Applied Physics, 2015. 117(4): p. 045702.

5. Chan, C.E., et al., Rapid stabilization of high-performance multicrystalline p-type silicon PERC cells. IEEE Journal of Photovoltaics, 2016. 6(6): p. 1473-1479.

6. Hamer, P., et al., Investigations on accelerated processes for the boron-oxygen defect in p-type Czochralski silicon. Solar Energy Materials and Solar Cells, 2016. 145: p. 440-446.

7. Payne, D., et al., Acceleration and mitigation of carrier - induced degradation in $p$ - type multi crystalline silicon. physica status solidi (RRL)-Rapid Research Letters, 2016. 10(3): p. 237-241.

8. Krauß, K., et al., Fast regeneration processes to avoid light-induced degradation in multicrystalline silicon solar cells. IEEE Journal of Photovoltaics, 2016. 6(6): p. 1427-1431.

9. Peral, A., et al., Impact of Extended Contact Cofiring on Multicrystalline Silicon Solar Cell Parameters. IEEE Journal of Photovoltaics, 2017. 7(1): p. 91-96.

10. Chan, C., et al., Instability of Increased Contact Resistance in Silicon Solar Cells Following Post Firing Thermal Processes. Solar RRL, 2017: p. 1700129.

11. Hamer, P., et al., Modelling of hydrogen transport in silicon solar cell structures under equilibrium conditions. Journal of Applied Physics, 2017.

12. Mäckel, H. and P.P. Altermatt, Current transport through lead-borosilicate interfacial glass layers at the screen-printed silver-silicon front contact. IEEE Journal of Photovoltaics, 2015. 5(4): p. 10341046.

13. Lin, C.-H., et al., Investigation of Ag-bulk/glassy-phase/Si heterostructures of printed Ag contacts on crystalline Si solar cells. Solar Energy Materials and Solar Cells, 2008. 92(9): p. 1011-1015.

14. Li, Z., L. Liang, and L. Cheng, Electron microscopy study of front-side Ag contact in crystalline Si solar cells. 2009, AIP.

15. Ballif, C., et al., Silver thick-film contacts on highly doped n-type silicon emitters: structural and electronic properties of the interface. Applied physics letters, 2003. 82(12): p. 1878-1880.

16. Hallam, B.J., et al., Method of Extracting Solar Cell Parameters From Derivatives of Dark I-V Curves. IEEE Journal of Photovoltaics, 2017. 7(5): p. 1304-1312.

17. Chan, D.S. and J.C. Phang, Analytical methods for the extraction of solar-cell single-and doublediode model parameters from IV characteristics. IEEE Transactions on Electron devices, 1987. 34(2): p. 286-293. 
18. Clugston, D.A. and P.A. Basore. PC1D version 5: 32-bit solar cell modeling on personal computers. in Photovoltaic Specialists Conference, 1997., Conference Record of the Twenty-Sixth IEEE. 1997. IEEE.

19. Reeves, G. and H. Harrison, Obtaining the specific contact resistance from transmission line model measurements. IEEE Electron device letters, 1982. 3(5): p. 111-113.

20. Dekkers, H., L. Carnel, and G. Beaucarne, Carrier trap passivation in multicrystalline Si solar cells by hydrogen from Si N x: H layers. Applied physics letters, 2006. 89(1): p. 013508.

21. Li, H., et al., POCl3 diffusion for industrial Si solar cell emitter formation. Frontiers in Energy, 2017. 11(1): p. 42-51.

22. Voronkov, V.V. and R. Falster, Formation, dissociation, and diffusion of various hydrogen dimers in silicon. physica status solidi (b), 2017.

23. Pearton, S., J. Corbett, and T. Shi, Hydrogen in crystalline semiconductors. Applied Physics A, 1987. 43(3): p. 153-195.

24. Sopori, B., et al., Hydrogen in silicon: a discussion of diffusion and passivation mechanisms. Solar Energy Materials and Solar Cells, 1996. 41: p. 159-169.

25. Chan, C., et al., Modulation of Carrier - Induced Defect Kinetics in Multi - Crystalline Silicon PERC Cells Through Dark Annealing. Solar RRL, 2017. 1(2).

26. Niewelt, T., et al., Understanding the light - induced degradation at elevated temperatures: Similarities between multicrystalline and floatzone $p$ - type silicon. Progress in Photovoltaics: Research and Applications.

27. Jensen, M.A., et al., Evolution of LeTID Defects in p-Type Multicrystalline Silicon During Degradation and Regeneration. IEEE Journal of Photovoltaics, 2017. 7(4): p. 980-987.

28. Bredemeier, D., D.C. Walter, and J. Schmidt, Possible Candidates for Impurities in mc-Si Wafers Responsible for Light-Induced Lifetime Degradation and Regeneration. Solar RRL, 2017: p. 1700159. 